Revue d'histoire de l'enfance « irrégulière »

Le Temps de l'histoire

9 | 2007

Violences et jeunesse

\title{
Moins coupables ? Perceptions de la jeunesse et enjeux socio-politiques dans le débat sur la peine capitale en Belgique
}

Jérôme de Brouwer

\author{
(2) OpenEdition \\ Journals \\ Édition électronique \\ URL : http://journals.openedition.org/rhei/2092 \\ DOI : 10.4000/rhei.2092 \\ ISBN : 978-2-7535-1648-9 \\ ISSN : $1777-540 \mathrm{X}$ \\ Éditeur \\ Presses universitaires de Rennes \\ Édition imprimée \\ Date de publication : 15 novembre 2007 \\ Pagination : 93-106 \\ ISSN : 1287-2431
}

Référence électronique

Jérôme de Brouwer, « Moins coupables ? Perceptions de la jeunesse et enjeux socio-politiques dans le débat sur la peine capitale en Belgique », Revue d'histoire de l'enfance « irrégulière » [En ligne], 9 | 2007, mis en ligne le 01 novembre 2009, consulté le 04 décembre 2020. URL : http:// journals.openedition.org/rhei/2092; DOI : https://doi.org/10.4000/rhei.2092 


\section{Moins coupables ? Perceptions de}

la jeunesse et enjeux socio-politiques

dans le débat sur la peine capitale en Belgique au XIXème siècle

La question qui occupe les parlementaires belges entre 1851 et 1853, au cœur des débats sur la réforme du Code pénal, a connu il y a peu une actualité pardelà l'Atlantique. Par son arrêt du 1er mars 2005, la plus haute juridiction des Etats-Unis, en la déclarant contraire au huitième amendement de la Constitution, a exclu l'application de la peine capitale aux mineurs de moins de 18 ans. ${ }^{(2)}$ Elle autorise enfin une nation désireuse de répandre les bienfaits de la civilisation à prendre ses distances par rapport aux quelques régimes autoritaires qui pratiquent encore ce genre d'exécutions. "Notre société considère que les mineurs sont moins coupables que le criminel d'âge mûr ", admettait Anthony Kennedy, l'un des juges de la Cour suprême. D’aucuns voient dans cette restriction du champ d'application de la peine capitale la manifestation d'une évolution lente mais continue des critères de décence (standards of decency) de la société américaine et un jalon vers l'abolition totale. ${ }^{(3)}$ Cette décision et les commentaires qu'elle suscite ne sont pas sans rappeler les débats qui prirent cours en Belgique au milieu du XIXème siècle, au moment où ce pays entendit se doter d'un nouveau code pénal.

Le projet de Code pénal présenté aux parlementaires belges en 1851 introduit le même type de restriction en son article 107 : l'abolition de la peine de mort pour les individus âgés de moins de 21 ans - âge de la majorité civile au moment des faits. Les moins de 21 ans sont considérés comme moins coupables même lorsqu'ils ont agi avec discernement. Cette extension partielle de la minorité civile vers le champ pénal paraît originale pour l'époque.(4) Elle marque une évolution par rapport au Code pénal de 1810, lequel avait déjà exclu l'application de la peine capitale pour les moins de 16 ans, que le crime ait été commis avec ou sans discernement. ${ }^{(5)}$
Jérôme de

Brouwer $^{(1)}$
(1) Assistant aux dépar-

tements d'Histoire et

de Droit de l'université catholique de Louvain, membre du Centre d'histoire du droit et de la justice. Préparation d'une thèse de doctorat consacrée à l'histoire de la peine de mort en Belgique au XIXème siècle.

(2) Arrêt Roper vs Simmons de la Cour suprême des États-Unis, 1er mars 2005.

(3) André Normandeau, «L'abolition de la peine de mort aux États-Unis... pour les jeunes de moins de 18 ans : un présage de l'abolition totale?", Revue pénitentiaire et de droit pénal, n² 2, 2005 , p. 371-374.

Jérôme de Brouwer / p. 93 à 106 
(4) Selon Julie Le

Quang Sang, la suppression de la peine de mort pour les mineurs [civils] serait intervenue en

France après l'accession au pouvoir de François

Mitterrand, un peu avant la loi du 9 octobre 1981 portant abolition de la peine de mort (Julie Le Quang Sang, La loi et le bourreau : La peine de mort en débats (I870-1985), Paris, L'Harmattan, 2001, p. 171). On peut regretter que l'auteure, dans la version publiée de sa thèse, se contente de cette seule mention, sans autre analyse.

(5) Code pénal de 1810 , art. 67 , prévoyant que la peine de mort serait remplacée par une peine de 10 à 20 ans d'emprisonnement en maison de correction. A fortiori, la peine de mort est exclue en ce qui concerne

le mineur de moins de 16 ans ayant commis un crime capital sans discer- nement. L'art. 66 du Code pénal de 1810 prévoit l'acquittement et la remise du mineur à ses parents ou l'envoi en maison de correction. Xavier Rousseaux, Marie-Sylvie Dupont-Bouchat, Claude Vael, [dir.], Révolutions et justices pénales en Europe : modèles français et traditions nationales, I780-I830, Paris-Montréal, L'Harmattan, 1999, 388 p.

(6) Jacques-Joseph Haus, Observations sur le projet de révision du Code pénal [...], Gand, 1835 , p. 213.

(7) Selon Adolphe Roussel, rapporteur de la commission de la Chambre, intervenant à propos de l'art. $107 \mathrm{du}$ projet de Code pénal (Annales parlementaires, Chambre des représentants, session I852-53, séance du 24 février 1853, p. 744 ).
Loin d'être anecdotique, la question de « l'âge d'admission à la peine de mort " dans le Code pénal belge renvoie aux représentations ambivalentes de la jeunesse dans l'esprit des élites politiques : entre fragilité et précocité dans le crime. ${ }^{(6)}$ Par ailleurs, l'enjeu de la consécration d'une cause d'excuse générale liée à l'imperfection des facultés, plus large que l'absence de discernement, tient dans le développement d'une abolition partielle et progressive de la peine de mort. Consacrer pareille cause d'excuse dans le Code pénal revient à restreindre le champ d'application ratione personae de la peine de mort. Cet objectif est assumé par les auteurs du projet de Code pénal et est conforme aux idées d'une partie des parlementaires. Reculer l'" âge d'admission à la peine de mort ", c'est restreindre le champ d'application de la peine capitale et répondre au vœu d'un code dont " on pût dire qu'il est un des codes les plus progressifs de l'Europe ». ${ }^{(7)}$ Malgré un consensus apparent sur l'idée d'une abolition progressive, la cause d'excuse prévue à l'article 107 du projet de Code pénal provoque une opposition très vive. La proposition de la commission de révision est combattue par ceux qui se représentent les jeunes criminels comme les auteurs des forfaits les plus terribles : parricide et régicide.

\section{Économie générale du projet de Code pénal belge et origine de l'article $107^{(8)}$}

Un premier projet de révision du Code pénal, largement inspiré de la loi française du 28 avril 1832, est présenté à la Chambre des représentants en 1834 par le ministre de la Justice Lebeau. Jugé incomplet, il n'est même pas discuté par les députés. Le criminaliste de renom qu'est Jacques-Joseph Haus publie dans la foulée, dès 1835, des Observations qui préfigurent les dis- 
positions du nouveau projet de Code pénal présenté à la Chambre en $1849 .^{(9)}$

Les motifs de la réforme du Code pénal sont clairement affichés. Pour les membres de la commission chargée d'élaborer le nouveau Code, la Belgique doit prendre ses distances par rapport au Code pénal de 1810 hérité du régime français, qui trouve sa source dans un pouvoir « arbitraire et ombrageux » et qui s'articule exclusivement - et erronément - autour de la notion d'utilité. La fierté qu'éprouvent les élites belges vis-à-vis de leur Constitution, envisagée comme une œuvre progressiste, ne peut s'accommoder d'un code trop sévère. ${ }^{(10)} \mathrm{La}$ jeune nation doit se doter de codes qui lui sont propres et qui traduisent sa vision politique. Si l'ensemble du projet traduit pour l'essentiel la pensée de Haus, lui-même ne fait pas mystère de sa filiation intellectuelle. Dès ses premières lignes, l'exposé des motifs fait expressément référence au père de l'École néo-classique, Pelegrino Rossi. ${ }^{(11)}$

En ce qui concerne la peine de mort, le projet répond aux principales critiques formulées contre le code de $1810 .{ }^{(12)}$ Considéré comme trop sévère en général, le Code pénal napoléonien voit la peine de mort comminée ${ }^{(13)}$ dans des cas jugés trop nombreux par le législateur de 1830 . Une restriction de fait du champ d'application de la peine capitale se systématise au lendemain de l'indépendance. En Belgique comme en France, la réforme de la législation pénale napoléonienne est précédée par l'extension de l'usage du droit de grâce. ${ }^{(14)}$ La pratique belge de la peine capitale renoue ainsi - et accentue une tendance apparue en Europe depuis la fin du XVIIIème siècle, qui tend à renvoyer le châtiment ultime à l'arrière-plan de l'arsenal pénal. ${ }^{(15)}$

De même que le Code pénal de 1810 et le Code pénal de 1791, le projet de Code pénal belge prévoit que le prévenu mineur de moins de 16 ans au moment des faits, ayant agi sans discernement, soit acquitté, mais, "d'après les circonstances, remis à ses parents ou conduit dans une maison de correction, pour y être élevé et détenu pendant le nombre d'années que le jugement déterminera, et qui toutefois ne pourra excéder l'époque où il aura accompli sa vingt et unième année ".(16) Il prévoit de même que le prévenu de moins de 16 ans accomplis ayant agi avec discernement, qui se serait rendu coupable d'un crime capital, soit condamné à une peine de huit à seize ans d'emprisonnement. ${ }^{(17)}$ Mais le projet innove en son article 107 :
(8) Le projet de révision de Code pénal a fait l'objet de plusieurs renumérotations au cours du processus législatif. Dans un souci de lisibilité, nous avons préféré conserver la numérotation initiale.

(9) Jacques-Joseph Haus, op. cit. Haus (1796-1881), d'origine bavaroise, catholique, est nommé professeur à l'université de Gand en 1817 par Guillaume Ier. Il sera recteur de l'université de Gand en 1865.

(10) Annales parlementaires, Chambre des représentants, session I849I850, Exposé des motifs ou rapports faits au nom de la commission du gouvernement, par M. J.J. Haus [...], p. 325 .

(11) Pelegrino Rossi (1787-1848), doyen de la faculté de droit de Paris, membre de l'Académie des sciences morales et poli- 


\begin{tabular}{|c|c|}
\hline tiques, pair de France. & Surveiller et punir. \\
\hline Privé de sa chaire par la & Naissance de la prison, \\
\hline révolution de 1848 , & Paris, Gallimard, 1975 ; \\
\hline il gagne les États pontifi- & Pieter Spierenburg, The \\
\hline caux, où il devient & Spectacle of Suffering. \\
\hline ministre de l'Intérieur et & Executions and the \\
\hline ministre des Finances & Evolution of Repression : \\
\hline \multirow[t]{2}{*}{ de Pie IX. } & from a Preindustrial \\
\hline & Metropolis to the European \\
\hline (12) Pierre Lascoumes, & Experience, Cambridge, \\
\hline Pierrette Poncela, Pierre & Cambridge University \\
\hline Lenoël, $A u$ nom de l'ordre. & Press, 1984 ; Victor A.C. \\
\hline Une histoire politique & Gatrell, The Hanging Tree. \\
\hline du Code pénal, Paris, & Execution and the English \\
\hline \multirow[t]{2}{*}{ Hachette, 1989, 404 p. } & People, 1770-1868, \\
\hline & Oxford, Oxford \\
\hline (13) NDLR : commi- & University Press, 1994 ; \\
\hline \multirow[t]{2}{*}{ nuer signifie minorer. } & Richard J. Evans, Rituals \\
\hline & of Retribution : Capital \\
\hline (14) En France, Louis & Punishment in Germany. \\
\hline XVIII renoue avec un & 1600-1880, Oxford, \\
\hline usage de la grâce moins & Oxford University Press, \\
\hline restrictif que la pratique & 1996 ; Martin Bergman, \\
\hline napoléonienne, dans & Dödsstraffet, kyrkan och \\
\hline l'attente de la réforme de & staten i Sverige fran $1700-$ \\
\hline 1832. Renée Martinage, & tal till rgoo-tal, Lund, \\
\hline Punir le crime. La répres- & 1996 ; Marie-Sylvie \\
\hline sion judiciaire depuis & Dupont-Bouchat, \\
\hline le Code pénal, Villeneuve- & La Belgique criminelle. \\
\hline d'Ascq, l'Espace juridique, & Droit, justice, société \\
\hline 1989, p. 277. & (XIVème-XXème siècles), \\
\hline & Louvain-la-Neuve, \\
\hline (15) Notamment : & Academia-Bruylant, 2006. \\
\hline
\end{tabular}

«La peine de mort ne sera prononcée contre aucun individu âgé de moins de vingt et un ans au moment du crime. Cette peine sera remplacée à l'égard des individus au-dessous de cet âge, par celle de la réclusion extraordinaire."

Cette dernière disposition est particulièrement originale par rapport à la filiation française. Rien de tel n'est prescrit dans la loi du 28 avril 1832 ni dans le projet Lebeau du 1er août 1834. Elle porte la marque de la philosophie générale qui sous-tend l'élaboration du projet de Code pénal, traversé par un souci d'humanité et par l'idée de progrès, en particulier à propos de la peine de mort. Pour les membres de la commission chargés de sa rédaction, la peine de mort ne doit être conservée que pour les attentats les plus graves envers les personnes, dans des cas limités pour lesquels il peut encore s'avérer utile et légitime de la maintenir, et dans l'attente de l'abolir complètement lorsque les mœurs auront évolué ou, en d'autres mots, lorsque le processus de civilisation aura gagné toutes les couches de la société. Selon Haus, principal inspirateur du projet, la peine de mort ne peut être abolie que par degrés. Tant qu'une punition plus appropriée ne peut la remplacer, elle doit être conservée, « son abolition totale et immédiate compromettrait gravement l'ordre social $»{ }^{(18)}$

D'une manière générale, le traitement judiciaire de la jeunesse ne paraît pas laisser Haus indifférent. " Nous pensons que le législateur doit à la jeunesse de plus grands ménagements ", précise-t-il dès 1835. Parmi ses commentaires, le professeur gantois soulève quelques propositions originales. Il exprime le souhait de voir la comparution des mineurs en cour d'assises exclue dans tous les cas. ${ }^{(19)}$ Il suggère également qu'aucun individu âgé de moins de 10 ans au moment des faits ne soit traduit en justice pour crime ou délit. ${ }^{(20)}$ Enfin, il propose d'in- 
sérer cette nouvelle disposition en vertu de laquelle la peine de mort serait remplacée par celle des travaux à perpétuité à l'égard des individus âgés de moins de 20 ans au moment du crime. Dans l'esprit de l'École néo-classique qui s'épanouit après 1830, soucieuse d'une moralisation de la loi pénale et d'une atténuation de sa rigueur, Haus se contente dans un premier temps de motiver son innovation par une préoccupation d'humanité. Il s'appuie sur la législation étrangère, les législations de Naples et de Parme qui défendent de prononcer la peine de mort contre des individus n'ayant pas atteint l'âge de 18 ans. Mais c'est dans la législation des États pontificaux, qui exclut l'application de la peine capitale pour les coupables de moins de 20 ans, que l'une des figures de la doctrine néo-classique, nourrie de pensée chrétienne, trouve son inspiration. Dans une représentation largement positive de la jeunesse, Haus s'explique :

« La vivacité des passions qui agitent la jeunesse ; l'absence, à cet âge, d'une perversité endurcie ; la certitude de parvenir à l'amendement de l'accusé ; tout commande à la société d'user d'indulgence envers de pareils coupables, et de ne pas les envoyer au supplice. "(21)

Cette proposition est reprise par la commission de révision en 1848, moyennant deux aménagements. Elle repousse jusqu'à 21 ans l'exclusion de la peine de mort et prévoit une autre peine de substitution. La peine des travaux forcés à perpétuité est remplacée par la réclusion à temps. Cette dernière modification ne constitue pas un renforcement du dispositif voulu par Haus. La peine des travaux forcés à perpétuité n'est pas réduite à la réclusion à temps dans le but de ménager davantage la jeunesse. Il faut y voir la conséquence de l'introduction dans le projet de Code pénal du modèle cellulaire. Suivant le système pennsylvanien, son article 22 prescrit l'isolement en cellules des condamnés, de jour comme de nuit. Pour les membres de la commission, ce bouleversement doit en entraîner un autre : la refonte de l'échelle des peines. La promotion de l'amendement véhiculée par le cellulaire doit nécessairement conduire à la suppression de l'enfermement perpétuel. Par ailleurs, la différence entre travaux forcés et réclusion leur paraît trop ténue dans le cadre du nouveau système. Dans le système en vigueur, chacune de ces peines emporte un travail pour le condamné, la peine de travaux forcés supposant seulement un labeur plus éprouvant. Les modalités du cellulaire leur semblent exclure
(16) Projet de Code pénal, art. 99.

(17) Projet de Code pénal, art. 100.

(18) Annales parlementaires, Chambre des représentants, Documents, session I849-50, séance du 14 décembre 1849 , Révision du titre préliminaire et du Ier livre $d u$ Code pénal, Exposé des motifs.

(19) Le projet Lebeau, à l'image de la loi française du 28 avril 1832, prévoyait en son article 68 que la comparution en assises ne serait exclue que pour autant que le mineur ne soit pas prévenu d'un crime passible d'une peine perpétuelle.

(20) Jacques-Joseph Haus, op. cit., p. 213.

(21) Jacques-Joseph Haus, op. cit., p. 214. 
(22) Annales parlementaires, Chambre des représentants, session I8495o, Exposé des motifs ou rapports faits au nom de la commission du gouvernement, par M. J.J. Haus [...], p. 328.

(23) Annales parlementaires, Chambre des représentants, Documents, session $1848-49$, p. 346.

(24) Annales parlementaires, Chambre des représentants, Documents, session I850-I85I, séance du 2 juillet 1851, Rapport fait au nom de la commission par M. Adolphe Roussel, p. 2136-2137. Adolphe Roussel (18091875), avocat, professeur à l'université de Louvain, ensuite à l'université libre de Bruxelles, député (1850-1854).

(25) François de Haussy (1789-1869), député libéral, ministre de la Justice de 1847 à 1850. cette différenciation. Les travaux forcés exclus, subsistent alors, outre la peine de mort, les peines de la réclusion et de la détention à temps. ${ }^{(22)}$

Sur le plan plus strictement juridique, la commission de révision, rappelant que "la résolution criminelle ou le dol se compose de deux éléments, la connaissance et la volonté ", estime que la jeunesse de l'accusé n'atténue pas sa culpabilité dans ce qui ressort de la connaissance. Sous le rapport de la volonté, par contre, elle voit dans la jeunesse une circonstance atténuante qui doit spécialement être définie par la loi, c'est-à-dire élevée au rang d'excuse. La commission entend sans doute prévenir les critiques de ceux qui verraient dans le système des circonstances atténuantes prévu par le projet Lebeau dans le sillage de la loi française du 28 avril 1832 encore une fois - une solution plus opportune. Elle reprend presque mot pour mot les propos de Haus, restreignant la peine capitale aux crimes les plus graves, "au degré le plus élevé de la perversité humaine ». Elle en écarte ceux qui ne paraissent pas encore gangrenés par le vice et le calcul criminel :

"Les passions vives de la jeunesse ne permettent pas au malfaiteur d'agir avec cette force de volonté, cette fermeté de résolution, avec cette réflexion calme, ce sang-froid qui, seul, mérite le plus sévère de tous les châtiments. » (23)

\section{Approbation à la Chambre des représentants, raidissement au Sénat}

Proposé à la discussion au mois de juillet 1851, l'article 107 est d'abord accueilli favorablement par les députés. Adolphe Roussel, rapporteur de la commission de la Chambre, admet :

«On voit clairement que les auteurs du projet, ne pouvant proposer la suppression complète du dernier supplice, ont fait des efforts heureux pour restreindre son application. Il est beau de concilier ainsi, par des mesures sages et prudentes, les besoins de l'ordre public et ceux de l'humanité ! »(24)

L'article 107 est approuvé sans la moindre opposition. On notera cependant que l'article proposé à la discussion n'est pas formulé dans les mêmes termes que dans le projet initial. Avant d'être soumis à la Chambre, le projet est passé entre les mains du ministre de la Justice. ${ }^{(25)}$ Celui-ci a manifesté son adhésion aux propositions de la commission, sous réserve d'un point important : la question des peines perpétuelles. Pour le ministre de la Justice, le maintien d'une peine perpétuelle conditionne la restriction du champ d'ap- 
plication de la peine de mort. Sa gravité constituerait pour le législateur une peine alternative à la peine de mort dans de nombreux cas. Le projet, amendé sur ce point, est ensuite soumis à une commission ad hoc au sein de la Chambre. Plus radicalement que le ministre de la Justice, les députés qui forment la commission rejettent la nouvelle échelle des peines dans son ensemble. Son rapporteur estime que la peine des travaux forcés doit être maintenue. La formulation de l'excuse générale de l'article 107 s'en trouve donc doublement modifiée. Elle est approuvée par les députés en ces termes :

"La peine de mort n'est prononcée contre aucun individu âgé de moins de vingt et un ans, au moment du crime. Elle est remplacée, contre les individus âgés de moins de vingt et un ans, par la peine des travaux forcés à perpétuité. » (26)

Quant à la restriction avouée du champ d'application de la peine de mort, elle ne doit pas faire illusion. La question du champ d'application doit être distinguée du débat sur le principe. En fait, le débat portant sur le maintien de la peine de mort ne déchaîne pas les passions, du côté de ses partisans comme de ses adversaires. Il apparaît d'emblée comme acquis. Il y a bien quelques parlementaires pour regretter sa conservation dans l'échelle des peines, mais un seul d'entre eux seulement pousse la conviction jusqu'à déposer une proposition formelle d'abolition. ${ }^{(27)}$ L'intérêt tout à fait relatif que suscite cette discussion se traduit par l'absence du quorum des présences requis pour procéder au vote. Le vote de la proposition est reporté à une séance ultérieure, en espérant que la question attire davantage les parlementaires dans l'hémicycle. Les députés, qu'ils soient de tendance libérale ou catholique, n'en demeurent pas moins très largement conservateurs, et l'opportunité de la peine capitale ne fait pas vraiment l'objet de débats. ${ }^{(28)}$ Seuls son champ d'application et ses modalités pratiques peuvent être discutés. Aucune voix ne se fait entendre sur l'article $107 \mathrm{du}$ projet. Les plus conservateurs ne prennent pas la peine de réagir, pour autant que le principe de la peine capitale soit maintenu.

Parvenu au Sénat, le projet de Code pénal subit d'autres assauts. Le maintien de la peine de mort y est plus évident encore qu'à la Chambre. L'article $7,1^{\circ}$, maintenant la peine de mort au sommet de l'échelle des peines, ne fait l'objet d'aucune discussion. ${ }^{(29)}$ Mais l'article 107 provoque au contraire une certaine agitation. Au sein de la commission du Sénat, le sénateur et ancien

(26) Article 99 du projet amendé par la commission de la Chambre.

(27) Annales parlementaires, Chambre des représentants, session I85I-52, séance du 14 novembre 1851, p. 48.

(28) C'est sans doute le cas jusqu'à la session 1866-1867, au cours de laquelle les débats parlementaires, relayés par une mobilisation de la presse libérale (abolitionniste) et catholique (en faveur du maintien de la peine capitale), prennent de l'ampleur, principalement sous l'impulsion du ministre de la Justice libéral Jules Bara.

(29) Annales parlementaires, Sénat, session I852-53, séance du 13 décembre 1852, p. 35. 
(30) Jules-Joseph

d'Anethan (1803-1888), député puis sénateur catholique, ministre de la Justice de 1843 à 1847.

(31) Charles Faider (1811-1893), ministre de la Justice de 1852 à 1855.

(32) Annales parlementaires, Sénat, session I852-I853, séance du 14 décembre 1852, p. 49. Guillaume Dorvillers, originaire de Couvin, avait été condamné à mort par arrêt de la cour d'assises de Namur du 26 février 1845 et exécuté à Namur le 18 avril 1845.

(33) Nicolas Defuisseaux (1802-1875), avocat, industriel, sénateur libéral. ministre catholique d'Anethan ${ }^{(30)}$ s'y était déjà vivement opposé. Il s'était montré assez persuasif pour que la commission suggère la suppression de l'article incriminé, ralliant le ministre de la Justice lui-même. ${ }^{(31)}$ N'avait-il pas agité le spectre du criminel le plus terrible, celui qui pouvait saper les fondements de l'ordre familial et social, le parricide ? D'Anethan avait ravivé le souvenir de Dorvillers, 17 ans, assassin de son père, guillotiné à Namur en 1845.(32)

Contrairement à la Chambre, une discussion assez vive s'engage en séance plénière. Le sénateur Defuisseaux souhaite son maintien, soulignant combien « il est vrai que la jeunesse est fragile et facile à entraîner parce qu'elle n’a pas la maturité nécessaire d'appréciation ». (33) D’Anethan ne désarme pas. Il admet bien que " la loi vient au secours de la fragilité humaine ", notamment quand un individu a moins de 16 ans, mais il ne peut admettre une disposition générale qui exclut la peine capitale pour tous les condamnés de moins de 21 ans. Il revient bientôt sur la question du parricide. Il fait valoir combien une telle disposition ébranlerait la notion de Justice, encouragerait le crime auprès de la jeunesse. Évoquant encore le parricide Dorvillers, il insiste sur le fait que « ce serait leur laisser supposer que jusqu'à une certaine limite d'âge, ils peuvent aller jusqu'à attenter, non pas impunément, mais du moins en ne risquant pas de porter leur tête sur l'échafaud, à la vie des auteurs de leurs jours ». D’une manière générale, pour l'ancien ministre de la Justice, le maintien de l'article 107 du projet conduirait à un péril pour la société en général et la sécurité des individus. Comme la plupart des parlementaires, comme la commission de révision, il pense que la peine de mort doit être maintenue pour les crimes les plus graves uniquement. Mais il rajoute qu'elle doit également trouver à s'appliquer " pour des coupables chez lesquels se montre une grande précocité dans le crime ». Il dénie la substance même de la réflexion de Haus et de la commission de révision en la matière. Leurs points de vue paraissent inconciliables. Le premier - Haus - voit dans la jeunesse la cause d'une altération de la volonté, donc du degré d'imputabilité ; le second, dans une conception pré-lombrosienne, y voit le caractère aggravant d'une disposition au crime. Cette impulsivité de la jeunesse qui, selon les plus progressistes, altère la résolution criminelle, est entendue par leurs détracteurs comme la source même du pire des crimes, la manifestation d'une criminalité primitive, irréductible. Et quel est le crime mis en avant par le sénateur d'Anethan ? 
Le crime ultime contre le père, précisément l'un de ces crimes que tous les parlementaires considèrent comme les plus atroces, l'un de ces crimes qui justifient le maintien provisoire du châtiment suprême : le parricide, mais aussi l'empoisonnement et l'assassinat, en particulier l'assassinat commis pour faciliter le vol. ${ }^{(34)}$ Il faut admettre avec Sylvie Lapalus la charge symbolique toute particulière qui affecte la figure du parricide, ce criminel qui, "dans une société qui valorise le modèle familial, [...] sape les principes essentiels de l'autorité ». ${ }^{(35)}$ Les héritiers du Code Napoléon, qui ont vu consacrer le primat de la puissance paternelle, ne sont-ils pas nourris d'une pensée de choix, celle de Maleville, l'un des quatre rédacteurs du Code civil, qui y voit le garant de « la conservation des mœurs et le maintien de la tranquillité publique " ? (36)

Cependant, la proposition d'amendement de d'Anethan ne rencontre pas l'adhésion d'une majorité de sénateurs. Le sénateur Defuisseaux, assisté par l'un ou l'autre collègue, défend efficacement le maintien de l'article 107. Il reproche surtout à d'Anethan de rechercher dans les annales judicaires des cas exceptionnels pour étayer son argumentation. Usant de l'arme statistique, le sénateur libéral s'applique aussi à démontrer que le crime le plus fréquent à cet âge est l'infanticide, crime pour lequel les parlementaires trouvent la peine de mort trop sévère ou inappropriée, quel que soit l'âge du prévenu. À une très courte majorité, par 17 voix contre 15, le maintien de l'article 107 est acquis. Du moins provisoirement, parce qu'une nouvelle occasion de débat se présente lorsque d'Anethan revient sur les articles du projet relatifs à la récidive et à la participation criminelle. Il parvient cette fois à convaincre ses collègues d'y apporter un amendement proposant l'inapplicabilité de l'article 107 en cas de récidive ou de concours de plusieurs crimes. En l'assortissant d'exceptions, l'ancien ministre de la Justice fait perdre à l'article 107 une part de son effectivité, et par là sa valeur de symbole.

\section{Réveil de la Chambre : opposition et solution de compromis}

Revenu auprès de la Chambre, selon l'usage de la procédure parlementaire, le projet amendé par le Sénat suscite cette fois des réactions nombreuses. L'article 107 n'y échappe pas, et sa suppression est bientôt envisagée ici aussi. Au nom de la cohérence de l'élaboration législative, le ministre de la Justice regrette de voir l'article 107 vidé de sa substance par une autre disposition. Il
(34) C'est ce qui ressort des débats à la Chambre et au Sénat, comme du texte d'Édouard Ducpétiaux, "Comptes de l'administration de la justice criminelle en Belgique (1826 à 1839) » in Bulletin de la Commission centrale de statistique, t. 1, Bruxelles, 1843 , p. 403-457.

(35) Sylvie Lapalus, La mort du vieux. Une histoire du parricide au XIXème siècle, Paris, Perrin, 2004, p. 526.

(36) Cité dans JeanPhilippe Lévy,

«L'évolution du droit familial français de 1789 au Code Napoléon ", in Irène Théry et Christian Biet, [dir.], La famille, la loi, l'État de la Révolution au Code civil, Paris, Imprimerie nationale, Centre Georges Pompidou, 1989, p. 511. 
(37) Annales parlementaires, Chambre des représentants, session I852-53, séance du 24 février 1853 , p. 743 .

(38) Colette Somerhausen, «L'évolution du droit des mineurs en Belgique ", in L'enfant. Quatrième partie : La délinquance juvénile, Recueils de la Société Jean Bodin pour l'histoire comparative des institutions, Bruxelles, 1977, p. 190. Voir également Jenneke Christiaens, De geboorte van de jeugddelinquent, Bruxelles, VUB Press, 1999, 430 p.

(39) Isidore Van Overloop (1814-1878), avocat, député puis sénateur catholique.

(40) Frédéric Chauvaud, De Pierre Rivière à Landru. La violence apprivoisée au XIXème siècle, s. 1., Brépols, 1991, p. 35. Voir aussi Axel Tixhon, Le préfere envisager sa suppression. Il n'y a pour ainsi dire que le rapporteur de la commission de la Chambre pour voler au secours de l'article 107. Adolphe Roussel rappelle la ratio legis d'un article qui « [...] forme une sorte de transaction entre les opinions diverses au sujet de la peine capitale : c'est l'acheminement vers la suppression de cette pénalité si terrible ». Dans la lignée de ce qu'avait exposé Haus, le rapporteur s'évertue à présenter les accusés de 18, 19 ou 20 ans comme des êtres encore proches de l'enfance, fondant une partie de son argumentation sur la comparaison avec la majorité civile, fixée à 21 ans. $^{(37)}$ Le ministre de la Justice ne semble pas encourager avec la même fougue la restriction du champ d'application de la peine capitale. Il paraît se satisfaire du système des circonstances atténuantes et de la grâce royale. Roussel s'y oppose. Il souligne l'importance du principe d'excuse, relevant des principes plus que de l'appréciation du cas d'espèce, plus aléatoire, à laquelle est soumis le système des circonstances atténuantes.

C'est autour de l'âge de la cristallisation des dispositions criminelles que s'articule le débat. Les députés s'attardent sur la limite d'âge proposée - 21 ans sur sa justification, et les intervenants multiplient les arguments pour la contester, pour trouver d'autres critères d'appréciation de la maturité, y compris dans le droit civil. D'une manière générale, dans la suite d'une législation révolutionnaire qui impose comme condition d'imputabilité la responsabilité morale de l'individu, résultat d'une volonté intelligente et libre, la question resurgit dans tous les débats relatifs à la délinquance juvénile. ${ }^{(38)}$ Le débat est d'autant plus récurrent qu'il s'agit de résoudre une équation sensible : définir un critère d’âge appliqué à la généralité des prévenus à une époque qui voit se développer un mouvement d'individualisation de la sanction pénale.

Sous l'apparente scientificité des interventions des parlementaires, la définition d'une catégorie d'âge relève d'une gymnastique périlleuse. Présentant une étude statistique soucieuse à l'extrême de classifications, un représentant catholique ${ }^{(39)}$ poursuit sur le rapport entre l'exemplarité des peines et l'âge de la maturité criminelle : «Si le but de toute loi pénale est de prévenir le crime par la crainte du châtiment, voyons à quel âge surtout la criminalité se développe... ", commencet-il. Partant d'un ratio de 100 crimes commis par les 21-25 ans, il note que les crimes commis par les 16-21 ans sont presque aussi nombreux que ceux commis par les 25-30 ans, et que leur nombre est plus important que ceux commis 
par les 35-40 ans. Et de faire remarquer par conséquent que, si le but de la loi pénale est d'effrayer, elle doit atteindre " précisément ceux qui ont l'âge auquel le crime se développe de la manière la plus complète, ceux qui arrivent à l'âge de 21 ans ». Catégorisation et recherche des "lois de la criminalité » avaient déjà conduit Adolphe Quetelet à démontrer que l'activité criminelle prenait un essor brutal à partir de 16 ans, pour ne cesser d'augmenter et atteindre son paroxysme vers 24 ans. ${ }^{(40)}$ Avec le même souci scientifique, l'un de ses coreligionnaires conservateurs remarque que "d'après les observations statistiques recueillies en divers pays, le penchant au crime est déjà très développé dans les jeunes gens de 16 à 21 ans [...] ». Il préconise donc de prémunir par l'exemple les individus de la catégorie considérée. ${ }^{(41)}$

Enfin, comme au Sénat, le parricide se presse une nouvelle fois dans l'hémicycle, nourrissant les interventions de plusieurs députés. Et à la suite de l'irruption insistante du parricide, presque naturellement, c'est le crime des régicides qui surgit dans le débat. Une actualité plus brûlante que celle de Dorvillers vient inspirer des députés : la tentative d'assassinat dont l'Empereur d'Autriche, François-Joseph, vient d'être victime. «Il y a peu de jours, entend-on à la Chambre, un souverain a manqué de tomber victime du poignard d'un assassin ; et quel est l'âge de l'auteur de cet infâme attentat? Si mes renseignements sont exacts, il n'a pas atteint l'âge de 21 ans. " ${ }^{(42)}$

Le rapporteur de la commission a beau faire valoir que le caractère exceptionnel d'un forfait ne doit pas influer sur l'action de ceux qui élaborent la loi pénale, il a beau rappeler l'origine de la disposition incriminée, et ses motifs, la boîte de Pandore est ouverte. Évoquant encore l'actualité, un député( ${ }^{(43)}$ insiste pour qu' " une modification soit apportée à cet article et que la punition contre des crimes abominables, comme le parricide et le régicide, ne soit pas telle qu'il soit permis de braver ainsi la justice des lois, qu'il soit permis surtout de se porter aux crimes les plus honteux, aux crimes les plus odieux en ayant pour soi une véritable impunité ». ${ }^{(44)}$

Lors de la séance suivante, le ministre de la Justice conclut que la Chambre peut revenir sur l'âge de 21 ans. Il souhaite cependant parvenir à un compromis qui concilie les opinions divergentes des députés et respecte le principe accepté lors du premier vote par l'assemblée. Il souhaite voir rejetées les exceptions introduites par le Sénat en cas de récidive et de participation cri- pouvoir des nombres. Une histoire de la production et de l'exploitation

des statistiques judiciaires belges (I795-I870), doctorat en philosophie et lettres (histoire), UCL, 2001, 974 p.

(41) Annales parlementaires, Chambre des représentants, session I85253, séance du 25 février 1853, p. 748 .

(42) Annales parlementaires, Chambre des représentants, session I852-53, séance du 24 février 1853, p. 743.

(43) Barthélémy

Dumortier, dit

Dumortier-Rutteau (1797-1878), député catholique.

(44) Annales parlementaires, Chambre des représentants, session I85253, séance du 24 février 1853, p. 743. 
(45) Annales parlemen-

taires, Chambre des représentants, session $1852-$ 53, séance du 25 février 1853, p. 747. Aux termes de l'article $144 \mathrm{du}$ Code civil, à 18 ans, l'homme peut se marier, devenir chef de famille, être appelé à avoir des enfants ; aux termes de l'article 374 du Code civil, l'homme, âgé de 18 ans, peut s'enrôler sans le consentement de ses parents ; aux termes de l'article $384 \mathrm{du}$ Code civil, tout citoyen âgé de 18 ans acquiert la jouissance de ses biens.

(46) Désiré de Haerne (1804-1890), abbé, membre du Congrès national (1830), député catholique, ensuite camérier secret du pape Pie IX et prélat domestique du pape Léon XIII.

(47) Annales parlementaires, Chambre des représentants, session I85253, séance du 25 février 1853, p. 749 . minelle, tout en reconnaissant les inquiétudes qui y ont été exprimées, ainsi qu'à la Chambre. Pour ce faire, il suggère de remplacer dans l'article 107 l'âge de 21 ans par l'âge de 18 ans. Il justifie cette nouvelle limite par le fait qu'elle s'apparente à l'une des majorités relatives consacrées dans les codes, principalement le Code civil. ${ }^{(45)}$

$\mathrm{Au}$ vu des données statistiques invoquées par les parlementaires, ce compromis semble avoir l'avantage de n'entraîner aucun impact effectif sur le champ d'application de la peine de mort, et donc aucune opposition, même si le parricide Dorvillers n'avait lui que 17 ans au moment de son crime. Le compromis semble pourtant satisfaire les deux parties, ceux qui s'accrochent à l'idée d'une restriction du champ d'application de la peine capitale et ceux qui craignent un affaiblissement de l'arsenal pénal et de l'exemplarité des peines vis-à-vis de la jeunesse. Dans l'argumentaire du ministre, le droit de grâce peut toujours venir suppléer aux insuffisances - toutes éventuelles - de l'article 107 amendé du projet.

\section{Conclusion : jeunesse et crime contre l'ordre politique et social}

L'amendement du ministre rallie enfin tous les députés, même si certains en profitent encore pour faire valoir leur opinion sur la peine capitale, comme l'abbé de Haerne, ${ }^{(46)}$ qui se perd dans une longue diatribe conservatrice traversée d'idées religieuses. L'article 77 du Code pénal promulgué en 1867 retiendra donc que " la peine de mort ne sera prononcée contre aucun individu âgé de moins de 18 ans accomplis au moment du crime. Elle sera remplacée par la peine des travaux forcés à perpétuité ».

La question des causes d'excuses liées à la jeunesse remue jusqu'au tréfonds le débat sur la peine capitale et soulève les peurs séculaires qui agitent les élites. L'intervention de l'abbé en témoigne de manière évidente :

«En présence de ce qui se passe, presque partout en Europe, ma raison recule devant des mesures trop brusques [...] Il semble que des passions terribles et féroces s'agitent plus que jamais dans les entrailles du corps social, et annoncent les tiraillements, les convulsions d'une lutte suprême entre le bien et le mal. " (47)

La filiation entre parricide et régicide dans le discours conservateur laisse distinctement apparaître la dimension sociopolitique du débat. Cinq ans à peine 
après la chute de la Monarchie de Juillet en France, à une époque marquée par la fragilité des systèmes politiques européens, il ne fait aucun doute qu'une bonne partie des parlementaires éprouvent de l'appréhension à l'idée d'adoucir le régime des peines. Les interventions qui ont lieu à la Chambre et au Sénat entre 1851 à 1853 révèlent les angoisses profondes des élites face au contexte international, marqué par des bouleversements politiques et sociaux : révolutions, attentats contre les souverains ou encore développement des revendications sociales. Au-delà de la permanence du parricide au sommet de la hiérarchie pénale, l'insistance avec laquelle le spectre du parricide et du régicide est introduit dans l'argumentaire de ceux qui élaborent la loi pénale manifeste au plus haut point leur crispation et leur angoisse devant l'éventualité d'un renversement de l'ordre établi, d'un bouleversement de leur système de valeurs politiques, sociales et familiales. La peur de ces bouleversements s'alimente d'une représentation de la jeunesse mue par " la vivacité des passions ", cette impulsivité qui devrait motiver au contraire, dans l'esprit des auteurs du projet, l'indulgence formulée à l'article 107.

Le crime juvénile qui préoccupe les parlementaires paraît être celui qui vise la filiation, d'une manière plus particulière le rapport à l'autorité et, sans doute, à la génération politique. La réaction si vive de certains parlementaires exprime-t-elle la crispation de la génération des pères d'une jeune nation à peine trentenaire ? "Les révolutions de 1848 [...], remarque Sergio Luzzato, mettent en lumière la fragilité d'un monde miné par la crise des hiérarchies en place. » ${ }^{(48)}$ Proche de l'un des épicentres révolutionnaires, le jeune État belge, sans voir son régime politique bouleversé, n'en a pas moins subi une fragilisation. Les années qui suivront verront s'articuler le raidissement et la nécessité de l'élargissement de la participation politique.

De façon exacerbée, l'argumentaire politique manifeste la part déterminante des angoisses sociopolitiques des élites au pouvoir, davantage que la matérialité de la criminalité, son impact réel et sa dangerosité. Face à une conception de la famille placée " au centre du dispositif disciplinaire ", ${ }^{(49)}$ pour ceux qui assument l'élaboration des lois, le crime le plus atroce est sans nul doute, non pas celui qui emporte une menace pour le plus grand nombre, non pas celui qui occupe le premier rang en termes quantitatifs, ${ }^{(50)}$ mais celui qui met directement en péril le groupe et le système politique et social qu'ils
(48) Sergio Luzzato,

" Jeunes révoltés et révolutionnaires (1789-1917)" in Histoire des jeunes en Occident. T2 : L'époque contemporaine, Paris, Seuil, 1996, p. 211.

(49) Sylvie Lapalus, "La famille assiégée de l'intérieur : jeunes parricides au XIXème siècle ", le Temps de l'histoire, $\mathrm{n}^{\circ} 3$, octobre 2000, p. 242.

(50) Dans son Essai sur la statistique morale de la France, Guerry classait le parricide au quinzième rang dans la série des crimes commis avant l'âge de 21 ans. Sylvie Lapalus, « La famille assiégée de l'intérieur..." ", op. cit., p. 237. 
(51) Michel Foucault, Moi Pierre Rivière, ayant égorgé ma mère, ma sæur et mon frère... Un cas de parricide au XIXème siècle, Paris, Gallimard-Julliard, coll. " Archives ", 1973 , p. 346.

(52) Pour une période ultérieure : Luisa Passerini, " La jeunesse comme métaphore du changement social. Deux débats sur les jeunes : l'Italie fasciste, l'Amérique des années 1950 " in Histoire des jeunes en Occident..., op. cit., p. 339-407. défendent. Si le modèle familial, qui est privilégié au cours de cette période, fait figure de " modèle rêvé d'une institution naturelle inégalitaire ", il légitime aussi le projet politique d'une élite. ${ }^{(51)}$ La jeunesse symbolise le bouleversement politique et social contre lequel les parlementaires, pères d'une jeune nation fragile, veulent mobiliser l'arsenal pénal. ${ }^{(52)}$ 